\title{
OPINIONES DE EXPERTOS PARA PROPUESTA A MODIFICACIÓN DEL ORAL HEALTH IMPACT PROFILE 49 EN ESPAÑOL
}

\section{MODIFICATION OF THE ORAL HEALTH IMPACT PROFILE 49 IN SPANISH AN EXPERT' S OPINION PROPOSAL}

\section{Ramírez Fernando ${ }^{1}$, Navarrete Diego², Quezada Camila², Vera Paula ${ }^{2}$}

\section{RESUMEN}

Objetivo: El Oral Health Impact Profile 49 en español (OHIP-49 sp), es un instrumento que se utiliza para evaluar la calidad de vida relacionada a salud oral. Este es un cuestionario que consta de 49 preguntas cualitativas.

Tras el estudio del instrumento OHIP-49 Sp se observó que carecía de una validación teórica, por lo que el objetivo principal fue identificar si es posible modificar el O-HIP $49 \mathrm{Sp}$ mediante la opinión de un comité de expertos, con el fin de aplicarse en adultos mayores.

Métodos: Para esto, se realizó un estudio descriptivo de corte transversal con un comité de experto compuesto por 8 profesionales con estudios en áreas geriátricas, odontogeriátricas, gerontológicas y/o experiencia en trabajo con adultos mayores.

Resultados: Se evaluó cada pregunta correspondiente al cuestionario OHIP-49 Sp, en donde se obtuvo que en 13 preguntas se contestaron "en duda", "desacuerdo" o "muy en desacuerdo" con la forma en que estas estaban formuladas. Finalmente 5 preguntas fueron seleccionadas como las peores formuladas y que por tanto se deberían modificar. Además, las preguntas se clasificaron según el tipo de crítica que recibieron, en donde varias de ellas obtuvieron más de un tipo de crítica, siendo la más frecuente la falta de claridad y ambigüedad.

Conclusión: Tras analizar el cuestionario OHIP-49 Sp, se observó que efectivamente hay oportunidades de modificación respecto a la encuesta original.
1. Universidad de Valparaíso, Escuela de Odontología, Valparaíso, Chile.

2. Atención Privada Odontológica, Chile.

Correspondencia:

Fernando Ramírez

Escuela de Odontología. Subida Leopoldo Carvallo \#211, Valparaíso, Chile. CP: 2360004 Correo electrónico:

fernando.ramirez@uv.cl

PALABRAS CLAVES:

Adulto mayor; Salud oral; Calidad de vida; Testimonio expertos

\section{KEYWORDS:}

Aged; Oral Health; Quality of life; Experts testimony. 


\section{ABSTRACT}

Objetives: The Oral Health Impact Profile 49 in Spanish (OHIP-49 sp) is an instrument used to evaluate the oral health related quality of life. This questionnaire consists of 49 quality related questions.

After studying the OHIP-49 Sp instrument, it came to attention that it lacked theoretical validation; because of this, the main objective was to assess the possibility of a modification for OHIP-49 $\mathrm{Sp}$ by means of an expert committee and to be applied in elderly patients.

Methods: a cross sectional descriptive study was done in which the expert committee was composed of 8 professionals with studies in geriatrics, odontogeriatrics, geronthology and/or experience with elderly patients.

Results: Every question of the OHIP-49 Sp questionnaire was evaluated, obtaining that 13 of the 49 questions were deemed doubtful by the experts or they considered to disagree or strongly disagree with the way in which they were redacted. In the end, 5 questions were considered to be the worst formulated and, thus, should be subject to modifications. In addition, each of the questions were classified according to the score they received, several of the questions had more than one criticism made towards them, the most frequent being "lack of clarity" and "ambiguity".

Conclusion: After analysis of the OHIP-49 Sp questionnaire, it was noted that indeed there is opportunity for changes to be made in respect of the original questionnaire.

\section{INTRODUCCIÓN}

A nivel mundial, la mala salud oral entre las personas mayores ha sido evidente en los altos niveles de pérdida de dientes, la experiencia de caries dental y las tasas de prevalencia de enfermedad periodontal, xerostomía y precáncer/cáncer oral. El impacto negativo de las malas condiciones orales en la calidad de vida de los adultos mayores es un importante problema de salud pública.

La Organización Mundial de la Salud recomienda que los países adopten ciertas estrategias para mejorar la salud bucal de las personas mayores. $^{1}$

Debido a la transición demográfica avanzada en Chile y a la baja natalidad, la población de adultos mayores se ha ido incrementando. ${ }^{2}$ Es por esto por lo que se deben adoptar ciertas estrategias para mejorar la salud bucal en adultos mayores, tener un mejor acceso a la salud, oportunidad y garantía. Además de realizar políticas, metas y objetivos mensurables para la salud bucal. ${ }^{1}$

Es por estas causas que las investigaciones deben converger hacia la calidad de vida de adultos mayores, principalmente asociada a la promoción y prevención de las enfermedades.

Para obtener un cuadro completo de un paciente, se debe complementar la información clínica con la información que entregan los tests psicométricos que miden calidad de vida.

Uno de los instrumentos más conocido para evaluar la calidad de vida relacionada a salud oral es el cuestionario Oral Health Impact Profile que mide aspectos que los individuos consideran de importancia para su calidad de vida, lo cual es relevante, ya que las mediciones sobre calidad de vida humanizan la atención de salud, al considerar las percepciones de los pacientes sobre la misma. ${ }^{3}$

El objetivo de este artículo se basa principalmente en describir las opiniones del comité de expertos respecto al instrumento OHIP-49 $\mathrm{Sp}$ con el fin de modificarse en un futuro, para así lograr por una parte datos fidedignos de índice de calidad de vida en salud oral, y por otra parte que la aplicación del instrumento sea más fácil y práctica tanto para evaluadores como para evaluados.

\section{MATERIALES Y MÉTODOS}

Se realizó un estudio descriptivo observacional de corte transversal. Los elementos para evaluar en esta investigación correspondieron a las 49 preguntas del cuestionario OHIP-49 Sp. 
Se contó con un comité de expertos constituidos por 8 profesionales con estudios en áreas geriátricas, odontogeriátricas, gerontológicas y/o experiencia en trabajo con adultos mayores. Los cuales son: médico geriatra, enfermera geriátrica, psicóloga psicométrica, fonoaudióloga Gerópolis, terapeuta ocupacional con estudios en gerontología, experta SENAMA, asistente social con experiencia en adultos mayores y odontólogo con experiencia en adultos mayores. Por lo tanto, se incluyeron en este estudio a profesionales con el título universitario, que enviaron su currículum abreviado y que presentaron estudios en áreas geriátricas con experiencias (curso, diplomado, magíster, doctorado). Se excluyó a aquellos profesionales que no hubiesen residido en Chile, debido al desconocimiento de la realidad social y cultural del país.

El comité de expertos fue una selección no aleatoria por conveniencia, según su disposición y voluntad para participar. Estos fueron contactados vía correo electrónico y se les proporcionó un consentimiento informado. Toda información fue únicamente utilizada para fines investigativos y se respetó el anonimato de los participantes. Para determinar el tamaño muestral se utilizó el método de Delphi.

El método de Delphi permitió estructurar un proceso de comunicación grupal que tiene como objetivo acercarse al consenso de un grupo de expertos una vez que estos han analizado y reflexionado en torno a un problema, obteniéndose juicios y opiniones fundadas. Una de sus fortalezas es no requerir presencialmente a los expertos. ${ }^{4}$ (Tabla I)

El instrumento OHIP-49 Sp presenta una estructura de 7 dimensiones, las cuales son: limitación funcional, dolor físico, malestar psicológico, discapacidad física, discapacidad psicológica, discapacidad social y minusvalía.

Se utilizaron 5 pasos para crear el OHIP-49 Sp (figura 1): el modelo conceptual, obtención de enunciados sobre impacto social, peso de los enunciados, confiabilidad del perfil de impacto de salud oral y validez del perfil de impacto de salud oral. ${ }^{5}$

\section{Tabla I: Características del Método Delphi}

\begin{tabular}{|c|c|}
\hline Proceso iterativo & $\begin{array}{l}\text { Los expertos deben emitir su } \\
\text { opinión o respuestas en } \\
\text { variadas ocasiones en las } \\
\text { diferentes rondas de pregunta, } \\
\text { así podrán reflexionar y } \\
\text { reconsiderar sus respuestas lo } \\
\text { que permitirá estabilizar sus } \\
\text { opiniones. }\end{array}$ \\
\hline Anonimato & $\begin{array}{l}\text { Ningún miembro del grupo } \\
\text { conoce a quien corresponde } \\
\text { una respuesta en particular, } \\
\text { sino que el control de la } \\
\text { comunicación está en manos } \\
\text { del grupo coordinador por lo } \\
\text { que no se establece una } \\
\text { participación directa entre los } \\
\text { expertos y así se evitan } \\
\text { influencias. }\end{array}$ \\
\hline $\begin{array}{l}\text { Retroalimentación } \\
\text { o feedback } \\
\text { controlado }\end{array}$ & $\begin{array}{l}\text { Una vez analizadas las } \\
\text { respuestas de los expertos por } \\
\text { parte del grupo coordinador es } \\
\text { permitida que la información } \\
\text { circule entre los expertos } \\
\text { pudiendo establecerse un } \\
\text { lenguaje común. }\end{array}$ \\
\hline $\begin{array}{l}\text { Respuesta } \\
\text { estadística del } \\
\text { grupo }\end{array}$ & $\begin{array}{l}\text { En caso de que se solicite una } \\
\text { estimación numérica como } \\
\text { respuesta, se maneja la } \\
\text { mediana de las respuestas } \\
\text { dadas por los expertos. }\end{array}$ \\
\hline
\end{tabular}


Figura 1: Pasos de creación del OHIP-49 Sp.

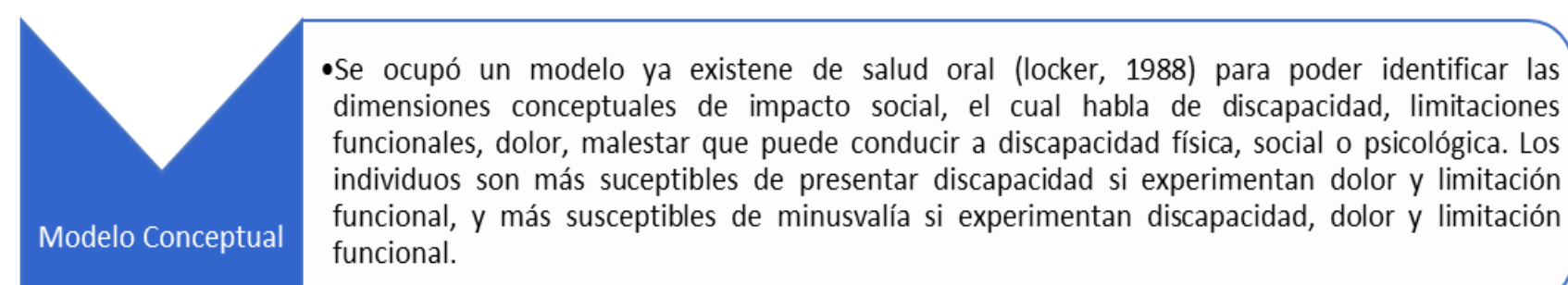

- Un conjunto de enunciados sobre impacto social fue recolectado durante entrevistas a un grupo de 64 pacientes odontológicos

Obtención de

enunciados sobre

impacto social

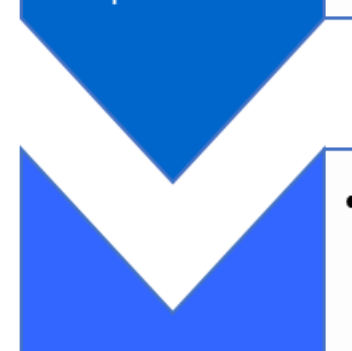

- Se obtuvo un set de 49 enunciados de los pacientes entrevistados y para darle peso al enunciado que refleje su relevancia se utilizó el método de Thurstone de comparaciones pareadas (Edwars, 1957). Ello se hizo para reflejar la importancia relativa de cada enunciado. Los resultados fueron convertidos a pesos numéricos usando los métodos descritos por Mckenna et al. (1981). El Peso del enunciado consenso entre los jurados fue determinado usando el método de Kendall, el cual refleja el nivel de acuerdo entre jueces.

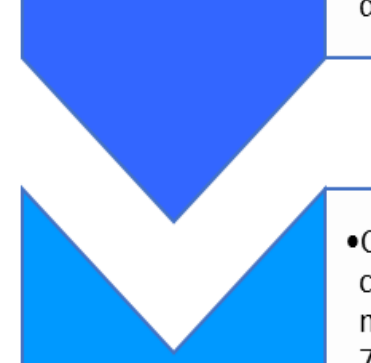

- Cada enunciado fue reestructurado como pregunta obteniéndose las 49 preguntas que contene el cuestionario y luego se entrevistó a una cohorte de personas de 60 años o más dividiéndose la muestra en igual cantidad de hombres y mujeres en los siguientes grupos de edad: 60-64, 65-69,

Confiabilidad del perfil de impacto de salud oral $70-74,75-79$ y de 80 años en adelante. La confiabilidad fue determinada por el coeficiente alfa de Cronbach; también se midió la estabilidad usando coeficiente de correlación intraclase preguntando el cuestionario a un sub-grupo del cohorte 3 meses después.

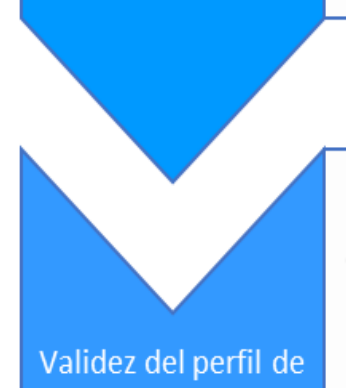

- Se determinó la validez convergente comparando las respuestas de OHIP del cohorte antes entrevistado, 20 meses después con las respuestas de un conjunto distinto de items de impacto social. 
Para el desarrollo de la investigación se explicó a los expertos participantes el procedimiento a seguir. Estos podían aceptar o rechazar la invitación y retirarse de la investigación cuando desearan. Una vez aprobada la solicitud, se envió al experto el consentimiento informado y el cuestionario.

Las opiniones del grupo de expertos fueron analizadas y resumidas, luego tabuladas en un conjunto de datos y analizadas estadísticamente, lo cual correspondió a la primera ronda. Se reenviaron a cada experto las opiniones del resto con respecto a las preguntas con peor valoración, que fueron 5 de las 49 preguntas que contenía el cuestionario. Esto permitió generar una retroalimentación a los expertos, quienes evaluaron estas preguntas $\mathrm{y}$ entregaron observaciones pertinentes. Las nuevas respuestas se tabularon en un conjunto de datos y se analizaron estadísticamente, lo que correspondió a la segunda ronda. Se observó la presencia de un consenso entre los expertos, por lo que el cuestionario se modificó en base a las opiniones sugeridas. Finalmente, se envió a cada experto el producto final modificado del cuestionario OHIP-49 Sp en donde no hubo objeción con respecto a la modificación, por lo que se obtuvo el consenso.

El análisis estadístico que se realizó en la primera ronda correspondió a la calificación de la opinión de cada integrante del comité respecto a las preguntas del cuestionario. Se colocó un puntaje en la escala Likert según el grado de acuerdo que se tuvo con la pregunta en base a la respuesta y se asignó un valor entre 1-5, donde 1 corresponde a que se percibió que el experto está muy en desacuerdo con la pregunta y 5 muy de acuerdo.

Cada valor de la escala de Likert fueron los siguientes:

- Muy en desacuerdo (1): Experto sugiere no añadir la pregunta al nuevo cuestionario.

- Desacuerdo (2): Experto sugiere reestructurar completamente la pregunta en el cuestionario ya sea por problemas en dimensión, forma de redacción o contenido, sin embargo, considera su mantención en el cuestionario modificado.

- En duda (3): Experto modifica parcialmente la pregunta ya sea en dimensión, forma o ambas, sin embargo, considera su mantención en el cuestionario modificado.

- Acuerdo (4): Experto modifica sutilmente la pregunta, ya sea cambiando un número acotado de palabras o eliminándolas.

- Muy de acuerdo (5): Experto concuerda totalmente con la pregunta y no presenta objeciones.

Luego, se calcularon medidas de tendencia central, específicamente moda y mediana, para cada una de las preguntas. Las preguntas cuyo puntaje obtuvieron una moda y/o mediana igual o inferior a 3, se enviaron nuevamente a los expertos para su reevaluación. Además, se establecieron 5 criterios respecto al tipo de crítica que se otorgó, los cuales fueron útiles para analizar el contenido de las respuestas entregadas. Estos criterios fueron la falta de claridad, presencia de ambigüedad, falta de neutralidad, falta de brevedad y presencia de carga emocional.

En la segunda ronda se envió a los expertos las 5 preguntas que obtuvieron una moda y/o mediana igual o inferior a 3 y las opiniones del resto de los integrantes del comité. Estos añadieron nuevas observaciones, las cuales se evaluaron nuevamente con una escala de Likert entre 1-5 y según el tipo de crítica. En esta segunda ronda hubo un mayor consenso entre los expertos, con lo cual se modificó el cuestionario y se les envió el producto final para que expresaran sus comentarios $u$ objeciones.

\section{RESULTADOS}

Luego de llegar al consenso de las respuestas en la primera ronda, se procedió a evaluarlas estadísticamente, en donde los 8 expertos en total promediaron que el 69,23\% está "muy de acuerdo" en las preguntas del OHIP-49 Sp. Se encontró que en 13 preguntas al menos 3 
Figura 2: Opinión de los 8 expertos según los consensos de las 49 preguntas del instrumento, destacando las 13 preguntas en que al menos 3 expertos contestaron "en duda", "desacuerdo" o "muy en desacuerdo".

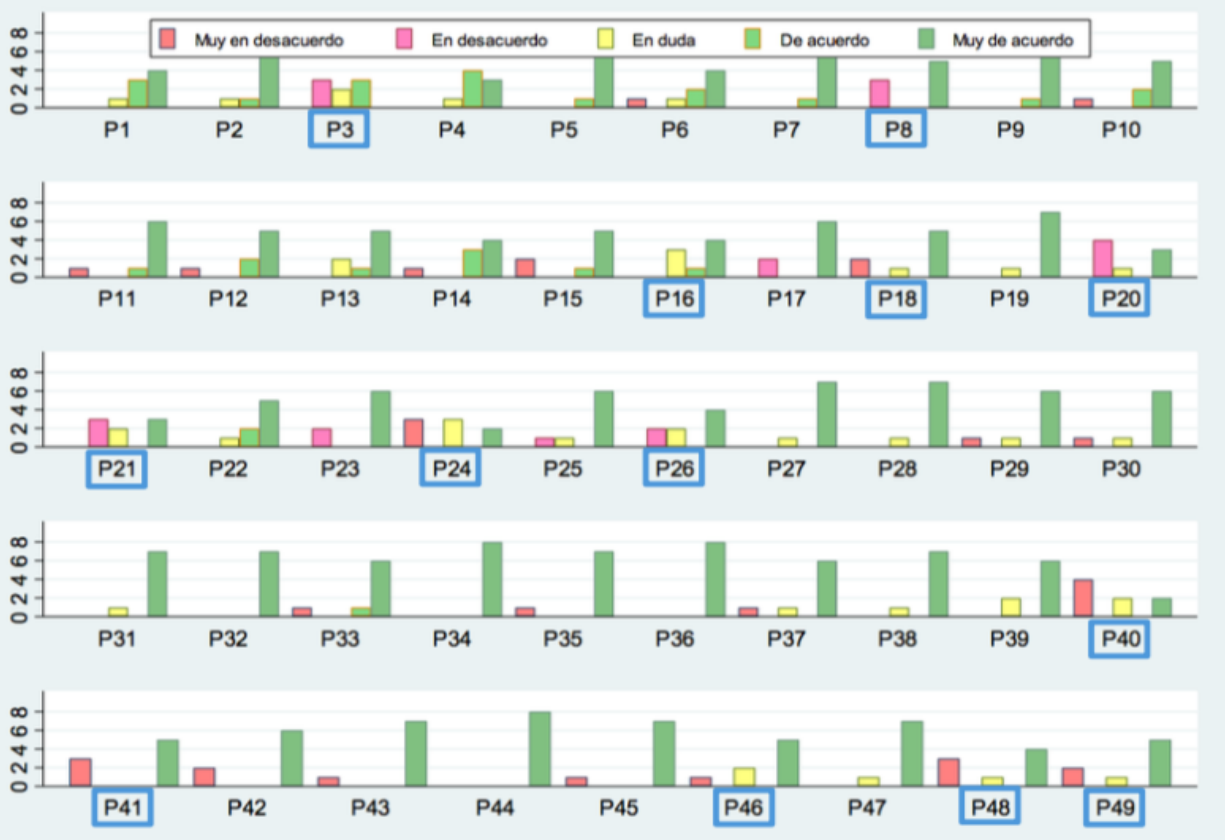

expertos contestaron "en duda", "desacuerdo" o "muy en desacuerdo" (Figura 2). Sin embargo, solo cinco se consideró deficientes, luego de evaluar moda y mediana de las preguntas en donde se tomó en consideración sólo aquellas con un puntaje igual o menor a 3 (Tabla II).

Además, se realizó un análisis con las 49 preguntas, en donde el 55,1\% presentaron una crítica a la falta de claridad. Se debe considerar que la mayoría de las preguntas obtuvieron más de un tipo de crítica.

Las siguientes preguntas fueron enviadas a los expertos para que expresaran sus acotaciones y/o modificaciones de estas:

1. Pregunta 3: ¿Ha notado que algún diente no se ve bien?

2. Pregunta 20: $¿$ Ha estado obsesionado/a por sus dientes, boca o prótesis?

3. Pregunta 21: ¿Los problemas dentales lo han hecho sentir totalmente infeliz?

4. Pregunta 24: ¿Ha sido poco clara la forma en que usted habla por problemas con sus dientes, boca o prótesis?

5. Pregunta 40: $¿$ Ha sido menos tolerante con su pareja o familia por problemas con sus dientes, boca o prótesis?

Al tener todos los datos se analizó el tipo de crítica contenida en la opinión de cada integrante del comité de expertos para cada una de las preguntas. Estas críticas se agruparon en 5 categorías para fines prácticos. Estas son: falta de claridad, falta de brevedad, presencia de ambigüedad, falta de neutralidad $y$ presencia de carga emocional.

A continuación, se presentan las críticas asociadas a cada pregunta y sus respectivas sugerencias de modificaciones:

- La pregunta 3 fue criticada por falta de claridad y ambigüedad. Se modificó a: ¿Ha notado que algún diente a cambiado de color, tamaño o posición?

- La pregunta 20 fue criticada por falta de claridad y presencia de carga emocional. Se modificó a: ¿Ha estado preocupado de sobremanera por su salud bucal?

- La pregunta 21 fue criticada por falta de neutralidad y presencia de carga emocional. Se modificó a: ¿Los problemas con sus dientes, boca o prótesis han afectado su estado de ánimo?

- La pregunta 24 fue criticada por falta de claridad. Se modificó a: ¿Ha tenido dificultades para hablar por problemas con sus dientes, boca o prótesis?

- La pregunta 40 fue criticada por falta de 
claridad, ambigüedad, neutralidad y presencia de carga emocional. Se modificó a: ¿Ha tenido dificultades con su pareja o familia por problemas con sus dientes, boca o prótesis?

Se comparó el comportamiento de cada integrante del comité durante el proceso y se logró observar que la desviación estándar fue elevada en un principio (1,39), pero al finalizar la experiencia disminuyó notoriamente $(0,58)$. Se obtuvo un delta del $50 \%$, por lo tanto, fue menos dispersa la opinión del comité. (Tabla III)

Los expertos sugirieron textualmente cómo reformular la pregunta y esta información era de conocimiento general. Por otra parte, todos coincidieron en que se debía reformular respecto a las críticas realizadas. Al momento de enviar la modificación de las preguntas, todos los expertos estuvieron de acuerdo con dicha modificación llegando a un consenso, pudiendo así modificar en un futuro la encuesta OHIP-49.

\section{DISCUSIÒN}

El principal hallazgo de este estudio es que al aplicar el cuestionario a los expertos y analizar sus respuestas con estadísticos de tendencia central, se concluye que es posible modificar el OHIP-49 Sp, con el fin de poder realizar un correcto uso del instrumento mucho más viable y confiable.

OHIP-49 Sp es un instrumento para medir la calidad de vida asociada a la salud oral, el cual el 2016 en la investigación de León ${ }^{7}$, se validó para la población de adultos mayores en Chile. En estudios epidemiológicos que han utilizado el OHIP, se ha encontrado que los dientes perdidos, la caries no tratada, la pérdida de la inserción periodontal y las barreras para el cuidado dental, están asociadas con un incremento en el impacto sobre el bienestar y la calidad de vida. ${ }^{3}$ Por esto, es esencial una correcta aplicación del instrumento de medición sobre la población, para evitar posibles sesgos al momento de aplicarse, lograr así claridad y
Tabla II: Preguntas que se enviaron a segunda ronda según moda y mediana

\begin{tabular}{|c|c|c|}
\hline $\mathbf{N}^{\mathbf{0}}$ de pregunta & Moda & Mediana \\
\hline 3 & 5 & 3 \\
\hline 20 & 1 & 2 \\
\hline 21 & 5 & 3 \\
\hline 24 & 3 & 3 \\
\hline 40 & 1 & 2 \\
\hline
\end{tabular}

Tabla III: Comparación de promedio de opinión de expertos según escala Likert

\begin{tabular}{|c|c|c|}
\hline Experto & $\begin{array}{c}\text { Promedio } \\
\text { primera } \\
\text { ronda }\end{array}$ & $\begin{array}{c}\text { Promedio } \\
\text { segunda } \\
\text { ronda }\end{array}$ \\
\hline $\mathbf{1}$ & 4,2 & 2,8 \\
\hline $\mathbf{2}$ & 5 & 3,6 \\
\hline $\mathbf{3}$ & 1 & 3,4 \\
\hline $\mathbf{4}$ & 1 & 2,6 \\
\hline $\mathbf{5}$ & 3 & 3 \\
\hline $\mathbf{6}$ & 3 & 4 \\
\hline $\mathbf{7}$ & 2,6 & 2,2 \\
\hline $\mathbf{8}$ & 2,6 & 3 \\
\hline Promedio & 2,8 & 3,08 \\
\hline general & & \\
\hline Desviación & 1,39 & 0,58 \\
\hline estándar & & 3,65 \\
\hline Prom + 1 Desv & 4,19 & 2,50 \\
\hline Prom - 1 Desv & 1,41 & \\
\hline
\end{tabular}

precisión de las preguntas a contestar, evitando posibilidad de interrogante respecto a falta de claridad, ambiguiedad o que simplemente no existiera comprensión de la pregunta, generando así una respuesta alterada respecto a lo que el instrumento quiere medir.

Dentro de las limitaciones está el tamaño 
muestral, cuyo número está lejos de ser representativo de todos los expertos en el área geriátrica y/o gerontológica. Además, el tipo de muestreo no es aleatorio, por lo que es posible que genera un sesgo de selección dentro de la investigación, lo que lleva a que tenga un menor peso estadístico. Por lo tanto, el comité de expertos en la investigación es selecto y no necesariamente representativo.

Otra limitación es el formato que se envía a los expertos para que contesten con respuestas abiertas, el cual posee un límite de palabras, lo que lleva a que no sea suficientemente flexible, impidiendo así una modificación total a su juicio con todo aquello que se estime conveniente. Además, la falta de claridad respecto a las preguntas, en donde algunos expertos se explayaron a través de respuestas meramente dicotómicas, lo que es no categorizable, por lo que hay pérdida de información y falta de datos que son relevantes para el objetivo planteado.

Es por esto, que se sugiere tener mayor número de integrantes en el comité de expertos y que sean escogidos de una manera aleatoria, para así poder modificar el instrumento con resultados estadísticamente significativos. Además, es necesario aplicar un estudio piloto con el objetivo de poner a prueba el instrumento modificado y así determinar su viabilidad de aplicación en la población.

$\mathrm{Si}$ bien el instrumento OHIP-49 Sp ha sido ampliamente estudiado, traducido y aplicado a diversas poblaciones en variados idiomas (6) y también ha sido utilizado para dar pie a versiones acortadas y/o cuestionarios que midan condiciones orales específicas, no se han encontrado en la literatura estudios que lo expongan a criterio de expertos de tipo multidisciplinario, por lo que la investigación sirve de base para un proyecto de modificación del OHIP-49 a gran escala.

\section{CONCLUSIÓN}

Tras someter el cuestionario OHIP-49 Sp a la opinión de un grupo de expertos, se observa que efectivamente hay oportunidad de modificación respecto a la encuesta original que facilita su uso en la población de adultos mayores en Valparaíso.

\section{CONFLICTOS DE INTERÉS}

Sin conflictos de interés

\section{REFERENCIAS}

[1] Petersen, P.E, Yanamoto, T, Dávila, R, González, A. Improving the oral health of older people: the approach of the WHO Global Oral Health Programme. Community Dent Oral Epidemiol. 2005;33(2): 81-92. DOI: 10.1111/j.1600-0528.2004.00219.x

[2] Ministerio de Salud, Estrategia Nacional de Salud Para el cumplimiento de los Objetivos Sanitarios de la Década 2011 -2022. Santiago. 2010. página 181, 185.

[3] Misrachi C, Espinoza I. Utilidad de las Mediciones de la Calidad de Vida Relacionada con la Salud. Revista Dental de Chile.2005;96(2): 28-36. URI: http://repositorio.uchile.cl/handle/2250/123542

[4] Varela-Ruiz M, Díaz-Bravo L, García-Durán R. Descripción y usos del método Delphi en investigaciones del área de la salud. Investigación educ. médica (revista digital). 2012 Jun; 1( 2 ): 90-95.

[5] Slade, G.D, Spencer, A.J. Development and evaluation of the Oral Health Impact Profile. Community Dent Health. 1994;11(1): 3-11. PMID: 8193981

[6] Awad M, Al-Shamrany M, Locker D, Allen F, Feine J. Effect of reducing the number of items of the Oral Health Impact Profile on responsiveness, validity and reliability in edentulous populations. Community Dent Oral Epidemiol. 2008;36(1):12-20. DOI: 10.1111/j.1600-0528.2006.00364.x

[7] León S, Bravo-Cavicchioli D, Giacaman R, Correa-Beltrán G, Albala C. Validation of the Spanish version of the oral health impact profile to assess an association between quality of life and oral health of elderly Chileans. Gerodontology. 2016 Mar;33(1):97-105. DOI: 10.1111/ger.12124.

\section{CÓMO CITAR ESTE ARTÍCULO}

Ramírez F, Navarrete D, Quezada C, Vera P. Opiniones de expertos para propuesta a modificación del oral health impact profile 49 en español. Appli. Sci. Dent. 2020; 1(1): 9-16 DOI: 10.22370/asd.2020.1.1.2108 